\title{
Upper limit on the cosmic-ray photon fraction at EeV energies from the Pierre Auger Observatory
}

The Pierre Auger Collaboration, J. Abraham ${ }^{g}$, P. Abreu ${ }^{\text {bk }}$, M. Aglietta ${ }^{\text {av }}$, C. Aguirre ${ }^{\text {k }}$, E.J. Ahn ${ }^{\text {bz }}$, D. Allard ${ }^{\text {aa }}$, I. Allekotte ${ }^{\mathrm{a}}$, J. Allen ${ }^{\mathrm{cc}}$, P. Allison ${ }^{\mathrm{ce}}$, J. Alvarez-Muñiz ${ }^{\mathrm{br}}$, M. Ambrosio aq, L. Anchordoqui ${ }^{\mathrm{co}}$, S. Andringa ${ }^{\mathrm{bk}}$, A. Anzalone au, C. Aramo aq, S. Argiró at, K. Arisaka ${ }^{\text {ch }}$, F. Arneodo ${ }^{\text {aw }}$, F. Arqueros ${ }^{\text {bo }}$, T. Asch ${ }^{\text {ah }}$, H. Asorey ${ }^{\text {a }}$, P. Assis ${ }^{\text {bk }}$, J. Aublin ${ }^{\text {ac }}$, M. Ave ${ }^{\text {ci }}$, G. Avila ${ }^{\mathrm{i}}$, T. Bäcker ${ }^{\mathrm{a}}$, D. Badagnani ${ }^{\mathrm{e}}$, K.B. Barber ${ }^{\mathrm{j}}$, A.F. Barbosa ${ }^{\mathrm{m}}$, S.L.C. Barroso ${ }^{\text {r }}$, B. Baughman ${ }^{\text {ce }}$, P. Bauleo ${ }^{\text {bx }}$, J.J. Beatty ${ }^{\text {ce }}$, T. Beau ${ }^{\text {aa }}$, B.R. Becker ${ }^{\text {cl }}$, K.H. Becker ${ }^{\text {af }}$,

A. Bellétoile ${ }^{\text {ad }}$, J.A. Bellido ${ }^{\mathrm{j}, \mathrm{cf}}$, S. BenZvi ${ }^{\mathrm{cn}}$, C. Berat $^{\text {ad }}$, P. Bernardini ${ }^{\text {ap }}$, X. Bertou ${ }^{\text {a }}$, P.L. Biermann ${ }^{\text {ai }}$, P. Billoir ${ }^{\text {ac }}$,

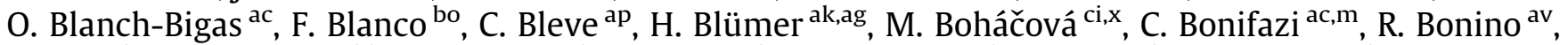
J. Brack $^{\text {bx }}$, P. Brogueira ${ }^{\text {bk }}$, W.C. Brown ${ }^{\text {by }}$, R. Bruijn ${ }^{\text {bt }}$, P. Buchholz ${ }^{\text {al }}$, A. Bueno ${ }^{\text {bq }}$, R.E. Burton ${ }^{\text {bv }}$, N.G. Busca ${ }^{\text {aa }}$, K.S. Caballero-Mora ${ }^{\text {ak }}$, L. Caramete ${ }^{\mathrm{ai}}$, R. Caruso $^{\text {as }}$, W. Carvalho ${ }^{\circ}$, A. Castellina $^{\text {av }}$, O. Catalano au, L. Cazon ${ }^{\text {ci, }}$, R. Cester ${ }^{\text {at }}$, J. Chauvin ${ }^{\text {ad }}$, A. Chiavassa ${ }^{\text {av }}$, J.A. Chinellato ${ }^{\mathrm{p}}$, A. Chou ${ }^{\text {bz,cc }}$, J. Chudoba ${ }^{\mathrm{x}}$, J. Chye $^{\text {cb }}$, R.W. Clay ${ }^{\mathrm{j}}$, E. Colombo $^{\mathrm{b}}$, R. Conceição ${ }^{\text {bk }}$, B. Connolly ${ }^{\mathrm{cm}}$, F. Contreras ${ }^{\mathrm{h}}$, J. Coppens ${ }^{\text {be,bg }}{ }^{\text {, A. Cordier }}{ }^{\mathrm{ab}}$, U. Cotti $^{\mathrm{bc}}$, S. Coutu ${ }^{\text {cf }}$, C.E. Covault ${ }^{b v}$, A. Creusot ${ }^{\mathrm{bm}}$, A. Criss ${ }^{\text {cf }}$, J. Cronin ${ }^{\mathrm{ci}}$, A. Curutiu ${ }^{\mathrm{a}}$, S. Dagoret-Campagne ${ }^{\mathrm{ab}}$, K. Daumiller ${ }^{\text {ag }}$, B.R. Dawson ${ }^{j}$, R.M. de Almeida ${ }^{\mathrm{p}}$, M. De Domenico ${ }^{\text {as }}$, C. De Donato ${ }^{\text {ao }}$, S.J. de Jong ${ }^{\text {be }}$, G. De La Vega ${ }^{g}$, W.J.M. de Mello Junior ${ }^{\mathrm{p}}$, J.R.T. de Mello Neto ${ }^{\mathrm{u}}$, I. De Mitri ${ }^{\mathrm{ap}}$, V. de Souza ${ }^{\mathrm{o}}$, G. Decerprit ${ }^{\text {aa }}$, L. del Peral ${ }^{\text {bp }}$, O. Deligny ${ }^{z}$, A. Della Selva ${ }^{\text {aq }}$, C. Delle Fratte ${ }^{a r}$, H. Dembinski ${ }^{\text {aj }}$, C. Di Giulio ${ }^{\text {ar }}$, J.C. Diaz ${ }^{\text {cb }}$, P.N. Diep ${ }^{c p}$, C. Dobrigkeit ${ }^{\text {p }}$, J.C. D’Olivo ${ }^{\text {bd }}$, P.N. Dong ${ }^{\mathrm{cp}}$, D. Dornic ${ }^{\mathrm{z}}$, A. Dorofeev ${ }^{\text {ca }}$, J.C. dos Anjos ${ }^{\mathrm{m}}$, M.T. Dova ${ }^{\mathrm{e}}$, D. D’Urso ${ }^{\mathrm{aq}}$, I. Dutan $^{\mathrm{ai}}$, M.A. DuVernois ${ }^{\mathrm{cj}}$, R. Engel ${ }^{\mathrm{ag}}$, M. Erdmann $^{\mathrm{aj}}$, C.O. Escobar $^{\mathrm{p}}$, A. Etchegoyen $^{\text {b }}$, P. Facal San Luis ${ }^{\text {ci,br }}{ }^{\text {, H. Falcke }}{ }^{\text {be,bh }}$, G. Farrar $^{c c}$, A.C. Fauth $^{\mathrm{p}}$, N. Fazzini ${ }^{\text {bz }}$, F. Ferrer ${ }^{\text {bv }}$,

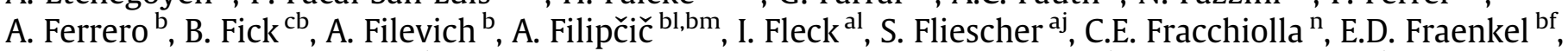
W. Fulgione ${ }^{\text {av }}$, R.F. Gamarra ${ }^{\text {b }}$, S. Gambetta ${ }^{\text {am }}$, B. García ${ }^{g}$, D. García Gámez ${ }^{\text {bq }}$, D. Garcia-Pinto ${ }^{\text {bo }}$, X. Garrido ag,ab ${ }^{\text {, G. Gelmini }}{ }^{\text {ch }}$, H. Gemmeke ${ }^{\text {ah }}$, P.L. Ghia ${ }^{\text {z,av }}$, U. Giaccari ${ }^{\text {ap }}$, M. Giller ${ }^{\text {bj }}$, H. Glass ${ }^{\text {bz }}$, L.M. Goggin ${ }^{\text {co, }}$, M.S. Gold ${ }^{\mathrm{cl}}$, G. Golup a , F. Gomez Albarracin e , M. Gómez Berisso a , P. Gonçalves ${ }^{\text {bk }}$, M. Gonçalves do Amaral v, D. Gonzalez ${ }^{\text {ak }}$, J.G. Gonzalez ${ }^{\text {bq,ca, D. Góra }}{ }^{\text {ak,bi }}$, A. Gorgi av, P. Gouffon ${ }^{\text {, }}$ S. Grebe ${ }^{\text {be,al }}$, M. Grigat ${ }^{\text {aj }}$, A.F. Grillo ${ }^{\text {aw }}$, Y. Guardincerri ${ }^{\text {d }}$, F. Guarino ${ }^{\text {aq }}$, G.P. Guedes ${ }^{\text {q }}$, J. Gutiérrez ${ }^{\text {bp }}$, J.D. Hague ${ }^{\text {cl }}$, V. Halenka ${ }^{\text {y }}$, P. Hansen ${ }^{\text {e }}$, D. Harari ${ }^{\text {a }}$, S. Harmsma ${ }^{\text {bf,bg }}{ }^{\text {, J.L. Harton }}{ }^{\text {bx }}$, A. Haungs ${ }^{\text {ag }}$, M.D. Healy ${ }^{\text {ch }}$, T. Hebbeker $^{\text {aj }}$, G. Hebrero ${ }^{\text {bp }}$, D. Heck ${ }^{\text {ag }}$, C. Hojvat $^{\text {bz }}$, V.C. Holmes $^{j}$, P. Homola bi ${ }^{\text {, J.R. Hörandel }}{ }^{\text {be }}$, A. Horneffer ${ }^{\text {be }}$, M. Hrabovský ${ }^{y, x}$, T. Huege $^{\text {ag }}$, M. Hussain ${ }^{\text {bm }}$, M. Iarlori ${ }^{\text {an }}$, A. Insolia ${ }^{\text {as }}$, F. Ionita $^{\text {ci }}$, A. Italiano $^{\text {as }}$, S. Jiraskova ${ }^{\text {be }}$, M. Kaducak ${ }^{\text {bz }}$, K.H. Kampert ${ }^{\text {af }}$, T. Karova ${ }^{x}$, P. Kasper ${ }^{\text {bz }}$, B. Kégl ${ }^{\text {ab }}$, B. Keilhauer ${ }^{\text {ag }}$, E. Kemp ${ }^{\text {p }}$, R.M. Kieckhafer ${ }^{\text {cb }}$, H.O. Klages ${ }^{\text {ag }}$, M. Kleifges ${ }^{\text {ah }}$, J. Kleinfeller ${ }^{\text {ag }}$, R. Knapik ${ }^{\text {bx }}$, J. Knapp ${ }^{\text {bt }}$, D.-H. Koang ${ }^{\text {ad }}$,

A. Krieger ${ }^{\text {b }}$, O. Krömer ${ }^{\text {ah }}$, D. Kruppke af , D. Kuempel af , N. Kunka ${ }^{\text {ah }}$, A. Kusenko ${ }^{\text {ch }}$, G. La Rosa ${ }^{\text {au }}$, C. Lachaud ${ }^{\text {aa }}$, B.L. Lago ${ }^{\mathrm{u}}$, M.S.A.B. Leão ${ }^{\mathrm{t}}$, D. Lebrun ${ }^{\mathrm{ad}}$, P. Lebrun $^{\mathrm{bz}}$, J. Lee ${ }^{\mathrm{ch}}$, M.A. Leigui de Oliveira $^{\mathrm{t}}$, A. Lemiere $^{\mathrm{z}}$, A. Letessier-Selvon ${ }^{\text {ac }}$, M. Leuthold ${ }^{\mathrm{aj}}$, I. Lhenry-Yvon ${ }^{\mathrm{z}}$, R. López ${ }^{\mathrm{ax}}$, A. Lopez Agüera $^{\mathrm{br}}$, J. Lozano Bahilo ${ }^{\mathrm{bq}}$, A. Lucero $^{\mathrm{av}}$, R. Luna García ay, M.C. Maccarone ${ }^{\mathrm{au}}$, C. Macolino $^{\text {an }}$, S. Maldera ${ }^{\mathrm{av}}$, D. Mandat $^{\mathrm{x}}$, P. Mantsch ${ }^{\mathrm{bz}}$, A.G. Mariazzi ${ }^{\text {e }}$, I.C. Maris ${ }^{\text {ak }}$, H.R. Marquez Falcon ${ }^{\text {bc }}$, D. Martello ${ }^{\text {ap }}$, J. Martínez ${ }^{\text {ay }}$, O. Martínez Bravo ${ }^{\text {ax }}$, H.J. Mathes ${ }^{\text {ag }}$, J. Matthews ${ }^{\text {ca,cg }}$, J.A.J. Matthews ${ }^{\text {cl }}$, G. Matthiae ${ }^{\text {ar }}$, D. Maurizio ${ }^{\text {at }}$, P.O. Mazur ${ }^{\text {bz }}$, M. McEwen ${ }^{\text {bp }}$, R.R. McNeil ${ }^{\text {ca }}$, G. Medina-Tanco ${ }^{\text {bd }}$, M. Melissas ${ }^{\text {ak }}$, D. Melo ${ }^{\text {at }}$, E. Menichetti $^{\text {at }}$, A. Menshikov $^{\text {ah }}$, R. Meyhandan ${ }^{\text {bf }}$, M.I. Micheletti ${ }^{\mathrm{b}}$, G. Miele ${ }^{\mathrm{aq}}$, W. Miller ${ }^{\mathrm{cl}}$, L. Miramonti ${ }^{\mathrm{ao}}$, S. Mollerach $^{\mathrm{a}}$, M. Monasor ${ }^{\text {bo }}$, D. Monnier Ragaigne ${ }^{a b}$, F. Montanet ${ }^{a d}$, B. Morales ${ }^{\text {bd }}$, C. Morello ${ }^{a v}$, J.C. Moreno $^{\mathrm{e}}$, C. Morris ${ }^{\text {ce }}$, M. Mostafá ${ }^{\mathrm{bx}}$, S. Mueller ${ }^{\text {ag }}$, M.A. Mueller ${ }^{\mathrm{p}}$, R. Mussa ${ }^{\text {at }}$, G. Navarra ${ }^{\text {av }}$, J.L. Navarro ${ }^{\text {bq }}$, S. Navas ${ }^{\text {bq }}$, P. Necesal ${ }^{\mathrm{x}}$, L. Nellen ${ }^{\text {bd }}$, 
C. Newman-Holmes ${ }^{\text {bz }}$, D. Newton ${ }^{\text {bt }}$, P.T. Nhung ${ }^{\mathrm{cp}}$, N. Nierstenhoefer ${ }^{\text {af }}$, D. Nitz ${ }^{\text {cb }}$, D. Nosek ${ }^{\mathrm{w}}$, L. Nožka $^{\mathrm{x}}$, J. Oehlschläger ${ }^{\mathrm{ag}}$, A. Olinto ${ }^{\mathrm{ci}}$, V.M. Olmos-Gilbaja ${ }^{\text {br }}$, M. Ortiz ${ }^{\text {bo }}$, F. Ortolani ${ }^{\mathrm{ar}}$, N. Pacheco ${ }^{\text {bp }}$,

D. Pakk Selmi-Dei ${ }^{\mathrm{p}}$, M. Palatka ${ }^{\mathrm{x}}$, J. Pallotta ${ }^{\mathrm{c}}$, G. Parente ${ }^{\mathrm{br}}$, E. Parizot ${ }^{\text {aa }}$, S. Parlati $^{\text {aw }}$, S. Pastor $^{\text {bn }}$, M. Patel $^{\text {bt }}$, T. Paul ${ }^{\text {cd }}$, V. Pavlidou ${ }^{\text {ci }}$, K. Payet ${ }^{\text {ad }}$, M. Pech ${ }^{\mathrm{x}}$, J. Pȩkala ${ }^{\text {bi }}$, R. Pelayo ${ }^{\text {bb }}$, I.M. Pepe ${ }^{\text {, }}$, . Perrone $^{\text {ap }}$, R. Pesce $^{\text {am }}$, E. Petermann ${ }^{c k}$, S. Petrera ${ }^{\text {an }}$, P. Petrinca ${ }^{a r}$, A. Petrolini ${ }^{a m}$, Y. Petrov ${ }^{\text {bx }}$, J. Petrovic $^{\text {bg }}$, C. Pfendner ${ }^{\text {cn }}$, A. Pichel $^{\mathrm{f}}$, R. Piegaia ${ }^{d}$, T. Pierog ${ }^{a g}$, M. Pimenta ${ }^{\text {bk }}$, T. Pinto ${ }^{\text {bn }}$, V. Pirronello ${ }^{\text {as }}$, O. Pisanti ${ }^{\text {aq }}$, M. Platino ${ }^{\text {b }}$, J. Pochon ${ }^{\text {a }}$, V.H. Ponce ${ }^{a}$, M. Pontz ${ }^{a l}$, P. Privitera ${ }^{c i}$, M. Prouza ${ }^{x}$, E.J. Quel ${ }^{c}, J_{\text {. Rautenberg }}{ }^{\text {a }}$, D. Ravignani ${ }^{b}$, A. Redondo ${ }^{\text {bp }}$, S. Reucroft ${ }^{\text {cd }}$, B. Revenu ${ }^{\text {ae }}$, F.A.S. Rezende ${ }^{\mathrm{m}}$, J. Ridky ${ }^{\mathrm{x}}$, S. Riggi ${ }^{\text {as }}$, M. Risse $^{\text {af,al }}$, C. Rivière ${ }^{\text {ad }}$, V. Rizi $^{\text {an }}$, C. Robledo ${ }^{a x}$, G. Rodriguez ${ }^{\text {ar }}$, J. Rodriguez Martino ${ }^{\text {as }}$, J. Rodriguez Rojo ${ }^{\text {h }}$, I. Rodriguez-Cabo ${ }^{\text {br }}$, M.D. Rodríguez-Frías ${ }^{\text {bp }}$, G. Ros ${ }^{\text {bo,bp }}$, J. Rosado ${ }^{\text {bo }}$, M. Roth ${ }^{\text {ag }}$, B. Rouillé-d'Orfeuil ${ }^{\text {aa }}$, E. Roulet ${ }^{a}$, A.C. Rovero ${ }^{\text {, }}$, F. Salamida an , H. Salazar ${ }^{\text {ax }}$, G. Salina ${ }^{\text {ar }}$, F. Sánchez ${ }^{\text {bd }}$, M. Santander ${ }^{\text {h }}$, C.E. Santo ${ }^{\text {bk }}$, E.M. Santos ${ }^{\text {u }}$, F. Sarazin ${ }^{\text {bw }}$, S. Sarkar ${ }^{\text {bs }}$, R. Sato ${ }^{\text {h }}$, N. Scharf ${ }^{\text {aj }}$, V. Scherini ${ }^{\text {af }}$, H. Schieler ${ }^{\text {ag }}$, P. Schiffer ${ }^{\text {aj }}$, A. Schmidt ${ }^{\text {ah }}$, F. Schmidt ${ }^{\text {ci, }}$ T. Schmidt ${ }^{\text {ak }}$, O. Scholten ${ }^{\text {bf }}$, H. Schoorlemmer ${ }^{\text {be,bg }}{ }^{\text {, J. Schovancova }}{ }^{\mathrm{x}}$, P. Schovánek ${ }^{\mathrm{x}}$, F. Schroeder ${ }^{\text {ag }}$, S. Schulte ${ }^{\text {aj }}$, F. Schüssler ${ }^{\text {ag }}$, D. Schuster ${ }^{\text {bw }}$, S.J. Sciutto ${ }^{\text {e }}$, M. Scuderi ${ }^{\text {as }}$, A. Segreto ${ }^{\text {au }}$, D. Semikoz $^{\text {aa }}$, M. Settimo ${ }^{\text {ap }}$, R.C. Shellard ${ }^{\mathrm{m}, \mathrm{n}}$, I. Sidelnik ${ }^{\mathrm{b}}$, B.B. Siffert ${ }^{\mathrm{u}}$, N. Smetniansky De Grande ${ }^{\mathrm{b}}$, A. Smiałkowski ${ }^{\text {bj }}$, R. Šmída ${ }^{x}$, B.E. Smith ${ }^{\text {bt }}$, G.R. Snow ${ }^{\text {ck }}$, P. Sommers ${ }^{\text {cf }}$, J. Sorokin ${ }^{j}$, H. Spinka ${ }^{\text {bu,bz }}$,

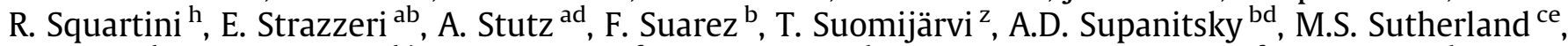
J. Swain ${ }^{\text {cd }}$, Z. Szadkowski bj, A. Tamashiro ${ }^{f}$, A. Tamburro ${ }^{\text {ak }}$, T. Tarutina ${ }^{\text {e }}$, O. Taşcau ${ }^{\text {af }}$, R. Tcaciuc $^{\text {al }}$, D. Tcherniakhovski ${ }^{\text {ah }}$, N.T. Thao ${ }^{\text {cp }}$, D. Thomas ${ }^{\text {bx }}$, R. Ticona ${ }^{1}$, J. Tiffenberg ${ }^{\text {d }}$, C. Timmermans ${ }^{\text {bg,be }}$, W. Tkaczyk ${ }^{\text {bj }}$, C.J. Todero Peixoto ${ }^{\mathrm{p}}$, B. Tomé ${ }^{\mathrm{bk}}$, A. Tonachini ${ }^{\text {at }}$, I. Torres ${ }^{\mathrm{ax}}$, P. Travnicek $^{\mathrm{x}}$, D.B. Tridapalli ${ }^{\mathrm{o}}$, G. Tristram ${ }^{\text {aa }}$, E. Trovato ${ }^{\text {as }}$, V. Tuci ${ }^{\text {ar }}$, M. Tueros ${ }^{\text {e }}$, R. Ulrich ${ }^{\text {ag }}$, M. Unger ${ }^{\text {ag }}$, M. Urban ${ }^{\text {ab }}$, J.F. Valdés Galicia ${ }^{\text {bd }}$, I. Valiño ${ }^{\text {br }}$, L. Valore ${ }^{\mathrm{aq}}$, A.M. van den Berg ${ }^{\text {bf }}$, R.A. Vázquez ${ }^{\text {br }}$, D. Veberič ${ }^{\mathrm{bm}, \mathrm{bl}}$, A. Velarde $^{\mathrm{l}}$, T. Venters ${ }^{\mathrm{ci}}$, V. Verzi ${ }^{\text {ar }}$, M. Videla ${ }^{\text {ca }}$, L. Villaseñor ${ }^{b c}$, S. Vorobiov ${ }^{\text {bm }}$, L. Voyvodic ${ }^{\text {bz }}$, H. Wahlberg ${ }^{\mathrm{e}}$, P. Wahrlich ${ }^{\mathrm{j}}$, O. Wainberg $^{\mathrm{b}}$, D. Warner ${ }^{\mathrm{bx}}$, A.A. Watson ${ }^{\text {bt }}$, S. Westerhoff ${ }^{\mathrm{cn}}$, B.J. Whelan ${ }^{\mathrm{j}}$, G. Wieczorek ${ }^{\text {bj }}$, L. Wiencke ${ }^{\text {bw }}$, B. Wilczyńska ${ }^{\text {bi }}$, H. Wilczyński ${ }^{\text {bi }}{ }^{\text {, C. Wileman }}{ }^{\text {bt }}$, M.G. Winnick ${ }^{\mathrm{j}}, \mathrm{H}$. Wu $^{\text {ab }}$, B. Wundheiler ${ }^{\text {b,ci }}{ }^{\text {, P. Younk }}{ }^{\text {bx }}$, G. Yuan ${ }^{\text {ca }}$, E. Zas ${ }^{\text {br }}$, D. Zavrtanik ${ }^{\text {bm,bl }}$, M. Zavrtanik ${ }^{\text {bl,bm }}$, I. Zaw ${ }^{c c}$, A. Zepeda ${ }^{\text {az,ba }}$, M. Ziolkowski ${ }^{\text {al }}$

\footnotetext{
${ }^{a}$ Centro Atómico Bariloche and Instituto Balseiro (CNEA-UNCUyo-CONICET), San Carlos de Bariloche, Argentina

${ }^{\mathrm{b}}$ Centro Atómico Constituyentes (Comisión Nacional de Energía Atómica/CONICET/UTN-FRBA), Buenos Aires, Argentina

${ }^{\mathrm{C}}$ Centro de Investigaciones en Láseres y Aplicaciones, CITEFA and CONICET, Argentina

${ }^{d}$ Departamento de Física, FCEyN, Universidad de Buenos Aires y CONICET, Argentina

e IFLP, Universidad Nacional de La Plata and CONICET, La Plata, Argentina

${ }^{\mathrm{f}}$ Instituto de Astronomía y Física del Espacio (CONICET), Buenos Aires, Argentina

${ }^{g}$ Observatorio Meteorologico Parque Gral. San Martin (UTN- FRM/CONICET/CNEA), Mendoza, Argentina

${ }^{\text {h }}$ Pierre Auger Southern Observatory, Malargüe, Argentina

i Pierre Auger Southern Observatory and Comisión Nacional de Energía Atómica, Malargüe, Argentina

${ }^{\mathrm{j}}$ University of Adelaide, Adelaide, S.A., Australia

${ }^{\mathrm{k}}$ Universidad Catolica de Bolivia, La Paz, Bolivia

${ }^{1}$ Universidad Mayor de San Andrés, Bolivia

${ }^{\mathrm{m}}$ Centro Brasileiro de Pesquisas Fisicas, Rio de Janeiro, RJ, Brazil

" Pontifícia Universidade Católica, Rio de Janeiro, RJ, Brazil

${ }^{\circ}$ Universidade de Sao Paulo, Instituto de Fisica, Sao Paulo, SP, Brazil

${ }^{\mathrm{P}}$ Universidade Estadual de Campinas, IFGW, Campinas, SP, Brazil

${ }^{\mathrm{q}}$ Universidade Estadual de Feira de Santana, Brazil

${ }^{\mathrm{r}}$ Universidade Estadual do Sudoeste da Bahia, Vitoria da Conquista, BA, Brazil

${ }^{\mathrm{s}}$ Universidade Federal da Bahia, Salvador, BA, Brazil

${ }^{\mathrm{t}}$ Universidade Federal do ABC, Santo André, SP, Brazil

${ }^{\mathrm{u}}$ Universidade Federal do Rio de Janeiro, Instituto de Física, Rio de Janeiro, RJ, Brazil

${ }^{\mathrm{v}}$ Universidade Federal Fluminense, Instituto de Fisica, Niterói, RJ, Brazil

${ }^{\mathrm{w}}$ Charles University, Faculty of Mathematics and Physics, Institute of Particle and Nuclear Physics, Prague, Czech Republic

${ }^{\mathrm{x}}$ Institute of Physics of the Academy of Sciences of the Czech Republic, Prague, Czech Republic

${ }^{y}$ Palacký University, Olomouc, Czech Republic

${ }^{\mathrm{z}}$ Institut de Physique Nucléaire d'Orsay (IPNO), Université Paris 11, CNRS-IN2P3, Orsay, France

aa Laboratoire AstroParticule et Cosmologie (APC), Université Paris 7, CNRS-IN2P3, Paris, France

${ }^{\mathrm{ab}}$ Laboratoire de l'Accélérateur Linéaire (LAL), Université Paris 11, CNRS-IN2P3, Orsay, France

${ }^{a}$ Laboratoire de Physique Nucléaire et de Hautes Energies (LPNHE), Universités Paris 6 et Paris 7, Paris Cedex 05, France

${ }^{a d}$ Laboratoire de Physique Subatomique et de Cosmologie (LPSC), Université Joseph Fourier, INPG, CNRS-IN2P3, Grenoble, France

ae SUBATECH, Nantes, France

af Bergische Universität Wuppertal, 42119 Wuppertal, Germany

${ }^{\text {ag }}$ Forschungszentrum Karlsruhe, Institut für Kernphysik, Karlsruhe, Germany

${ }^{\text {ah }}$ Forschungszentrum Karlsruhe, Institut für Prozessdatenverarbeitung und Elektronik, Germany

ai Max-Planck-Institut für Radioastronomie, Bonn, Germany

${ }^{\text {aj }}$ RWTH Aachen University, III. Physikalisches Institut A, Aachen, Germany

${ }^{\mathrm{ak}}$ Universität Karlsruhe (TH), Institut für Experimentelle Kernphysik (IEKP), Karlsruhe, Germany

${ }^{\text {al } U n i v e r s i t a ̈ t ~ S i e g e n, ~ S i e g e n, ~ G e r m a n y ~}$

${ }^{\mathrm{am}}$ Dipartimento di Fisica dell'Università and INFN, Genova, Italy
} 
${ }^{\text {an } U n i v e r s i t a ̀ ~ d e l l ' A q u i l a ~ a n d ~ I N F N, ~ L ' A q u i l a, ~ I t a l y ~}$

ao Università di Milano and Sezione INFN, Milan, Italy

${ }^{\mathrm{ap}}$ Dipartimento di Fisica dell'Università del Salento and Sezione INFN, Lecce, Italy

aq Università di Napoli "Federico II" and Sezione INFN, Napoli, Italy

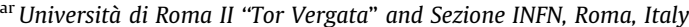

as Università di Catania and Sezione INFN, Catania, Italy

${ }^{\text {at } U n i v e r s i t a ̀ ~ d i ~ T o r i n o ~ a n d ~ S e z i o n e ~ I N F N, ~ T o r i n o, ~ I t a l y ~}$

au Istituto di Astrofisica Spaziale e Fisica Cosmica di Palermo (INAF), Palermo, Italy

av Istituto di Fisica dello Spazio Interplanetario (INAF), Università di Torino and Sezione INFN, Torino, Italy

aw INFN, Laboratori Nazionali del Gran Sasso, Assergi (L'Aquila), Italy

${ }^{a x}$ Benemérita Universidad Autónoma de Puebla, Puebla, Mexico

ay Centro de Investigacion en Computo del IPN, México, D.F., Mexico

${ }^{\mathrm{az}}$ Centro de Investigación y de Estudios Avanzados del IPN (CINVESTAV), México, D.F., Mexico

ba Instituto Nacional de Astrofisica, Optica y Electronica, Tonantzintla, Puebla, Mexico

${ }^{\mathrm{bb}}$ Unidad Profesional Interdisciplinaria de Ingenieria y Tecnologia Avanzadas del IPN, Mexico, D.F., Mexico

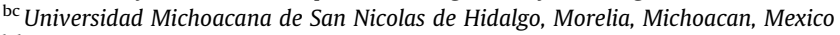

${ }^{\text {bd }}$ Universidad Nacional Autonoma de Mexico, Mexico, D.F., Mexico

be IMAPP, Radboud University, Nijmegen, Netherlands

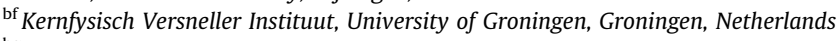

bg NIKHEF, Amsterdam, Netherlands

${ }^{b h}$ ASTRON, Dwingeloo, Netherlands

${ }^{\mathrm{bi}}$ Institute of Nuclear Physics PAN, Krakow, Poland

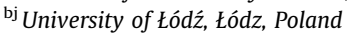

${ }^{\mathrm{bk}}$ LIP and Instituto Superior Técnico, Lisboa, Portugal

bl J. Stefan Institute, Ljubljana, Slovenia

${ }^{b m}$ Laboratory for Astroparticle Physics, University of Nova Gorica, Slovenia

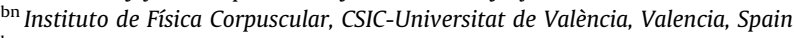

bo Universidad Complutense de Madrid, Madrid, Spain

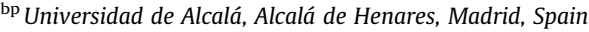

${ }^{\text {bq } U n i v e r s i d a d ~ d e ~ G r a n a d a ~ \& ~ C . A . F . P . E ., ~ G r a n a d a, ~ S p a i n ~}$

${ }^{\mathrm{br}}$ Universidad de Santiago de Compostela, Spain

${ }^{\text {bs }}$ Rudolf Peierls Centre for Theoretical Physics, University of Oxford, Oxford, United Kingdom

${ }^{b}$ School of Physics and Astronomy, University of Leeds, United Kingdom

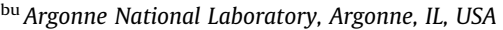

${ }^{\text {bv }}$ Case Western Reserve University, Cleveland, OH, USA

bw Colorado School of Mines, Golden, CO, USA

${ }^{\mathrm{bx}}$ Colorado State University, Fort Collins, CO, USA

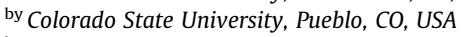

${ }^{\mathrm{bz}}$ Fermilab, Batavia, IL, USA

ca Louisiana State University, Baton Rouge, LA, USA

${ }^{\mathrm{cb}}$ Michigan Technological University, Houghton, MI, USA

${ }^{\mathrm{cc}}$ New York University, New York, NY, USA

${ }^{\mathrm{cd}}$ Northeastern University, Boston, MA, USA

ce Ohio State University, Columbus, OH, USA

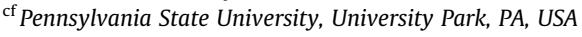

${ }^{\mathrm{cg}}$ Southern University, Baton Rouge, LA, USA

ch University of California, Los Angeles, CA, USA

ci University of Chicago, Enrico Fermi Institute, Chicago, IL, USA

${ }^{\mathrm{cj}}$ University of Hawaii, Honolulu, HI, USA

${ }^{\mathrm{ck}}$ University of Nebraska, Lincoln, NE, USA

${ }^{\mathrm{cl}}$ University of New Mexico, Albuquerque, NM, USA

$\mathrm{cm}$ University of Pennsylvania, Philadelphia, PA, USA

${ }^{\mathrm{cn}}$ University of Wisconsin, Madison, WI, USA

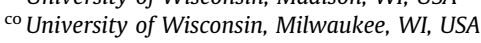

${ }^{\mathrm{cp}}$ Institute for Nuclear Science and Technology (INST), Hanoi, Viet Nam

\section{A R T I C L E I N F O}

Article history:

Received 4 March 2009

Received in revised form 8 April 2009

Accepted 18 April 2009

Available online 3 May 2009

\section{Introduction}

Data taken at the Pierre Auger Observatory were searched previously for ultra-high energy (UHE) photons above $10 \mathrm{EeV}$ [1,2]. In Ref. [1], the depth of shower maximum $X_{\max }$ of air showers observed by fluorescence telescopes in hybrid mode (i.e. with additional timing information from the ground array)

\begin{abstract}
A B S T R A C T
From direct observations of the longitudinal development of ultra-high energy air showers performed with the Pierre Auger Observatory, upper limits of 3.8\%, 2.4\%, 3.5\% and $11.7 \%$ (at $95 \%$ c.l.) are obtained on the fraction of cosmic-ray photons above $2,3,5$ and $10 \mathrm{EeV}\left(1 \mathrm{EeV} \equiv 10^{18} \mathrm{eV}\right)$, respectively. These are the first experimental limits on ultra-high energy photons at energies below $10 \mathrm{EeV}$. The results complement previous constraints on top-down models from array data and they reduce systematic uncertainties in the interpretation of shower data in terms of primary flux, nuclear composition and protonair cross-section.
\end{abstract}

(c) 2009 Elsevier B.V. All rights reserved. was used to place an upper limit of $16 \%$ on the photon fraction above $10 \mathrm{EeV}$, confirming and improving on previous limits from ground arrays [3-6]. In Ref. [2], the larger number of events taken with the Auger ground array alone allowed us to place a limit of $2 \%$ above $10 \mathrm{EeV}$, which imposes severe constraints on "top-down" models for the origin of ultra-high energy cosmic rays. 
Observations in hybrid mode are also possible at energies below $10 \mathrm{EeV}$. Decreasing the energy threshold increases the event statistics, which to some extent balances the factor $\sim 10$ smaller duty cycle compared to observations with the ground array alone. Thus, based on the previous work, the search for photons is now extended to lower energy (here down to $2 \mathrm{EeV}$ ). We also improve on our previous (statistics-limited) bound above $10 \mathrm{EeV}$ from Ref. [1].

Photons at EeV energies are expected to be produced in our cosmological neighborhood, as the energy attenuation length of such photons is only of the order of a few Mpc. Possible sources of EeV photons are the standard GZK process (see e.g. Refs. [7-9]), the production by nuclei in regions of intense star light (e.g. in the galactic center [10]), or exotic scenarios such as top-down models (see Ref. [11] for a review). Compared to our previous constraints on top-down models from Ref. [2], the bounds derived in this work provide a test of model predictions in a different energy range and using a different experimental technique, thus giving an independent confirmation of the model constraints.

Limits on EeV photons reduce corresponding systematic uncertainties in other analyses of air shower data. For instance, the presence of a substantial photon component can severely affect the reconstruction of the energy spectrum [12], the derivation of the proton-air cross-section $[13,14]$, and the interpretation of the observed average $X_{\max }$ [15] in terms of a nuclear primary composition.

The structure of the paper is as follows. In Section 2, the analysis is described and applied to the data. The results are discussed in Section 3.

\section{Data and analysis}

The present analysis follows closely the one described in detail in Ref. [1] which is called Hybrid-1 below. The basic idea is to compare the measured $X_{\max }$ values to those expected for primary photons, because UHE photon showers have significantly deeper average $X_{\max }$. We provide a summary of the analysis method, paying special attention to differences or changes in the approach compared to Hybrid-1.

The data used here were taken with a total of 18 fluorescence telescopes located at three sites ("Los Leones","Los Morados" and "Coihueco") between 1 December 2004 and 31 December 2007. The number of ground stations grew in this period from about 530 to 1450 . Compared to Hybrid-1 the data set above $10 \mathrm{EeV}$ increased in size by a factor $\sim 2.2$.

The event reconstruction [16] is based on an end-to-end calibration of the fluorescence telescopes [17], monthly models for the atmosphere [18], and an average aerosol model based on local atmospheric measurements [19]. The reconstruction of the longitudinal profile is described in [20]. A correction of $\sim 1 \%$ for the missing energy (energy carried by neutrinos or high energy muons) is applied to the reconstructed calorimetric energy, corresponding to the effective energy of primary photons [21].

The following quality cuts are applied to the collected events:

- number of phototubes in the fluorescence telescope triggered by the shower $\geqslant 6$;

- distance of closest approach of the reconstructed shower axis to the surface detector station with the largest signal is $<1.5 \mathrm{~km}$, and difference between the reconstructed shower front arrival time at this station and the measured tank time is $<300 \mathrm{~ns}$;

- normalized $\chi_{\text {prof }}^{2}$ of the longitudinal shower profile fit $[20]<6$, and ratio of $\chi_{\text {prof }}^{2}$ to $\chi_{\text {line }}^{2}<0.9$, where $\chi_{\text {line }}^{2}$ refers to a straight line fit (the latter cut essentially rejects profiles with too few data points);
- depth of shower maximum $X_{\max }$ observed in the telescope field of view (this cut may be relaxed in future to allow also the search for deeply penetrating events with $X_{\max }$ beyond the field of view);

- minimum angle between the viewing direction of a triggered pixel and the shower axis $>15^{\circ}$ (to reject events with a large Cherenkov light contamination);

- primary energy $E>f \cdot \mathrm{EeV}, f=2,3,5,10$ (the analysis in Hybrid1 was restricted to $f=10$ ).

The criterion of $X_{\max }$ being observed can introduce a bias against the deeply penetrating photon primaries (e.g. for near-vertical events). To reduce the dependence of the detector acceptance on composition, fiducial volume cuts are applied:

- shower zenith angle $>35^{\circ}+g_{1}(E)$

$$
g_{1}(E)= \begin{cases}10^{\circ}(\lg E / \mathrm{eV}-19.0) & \text { for } \lg E / \mathrm{eV} \leqslant 19.7 \\ 7^{\circ} & \text { for } \lg E / \mathrm{eV}>19.7\end{cases}
$$

- distance of telescope to shower core $<24 \mathrm{~km}+g_{2}(E)$

$$
g_{2}(E)= \begin{cases}12(\lg E / \mathrm{eV}-19.0) \mathrm{km} & \text { for } \lg E / \mathrm{eV} \geqslant 19.0 \\ 6(\lg E / \mathrm{eV}-19.0) \mathrm{km} & \text { for } \lg E / \mathrm{eV}<19.0\end{cases}
$$

The described cuts are identical to those from Hybrid-1 for showers $>10 \mathrm{EeV}$, but allow now for an extension of the energy range down to $2 \mathrm{EeV}$.

To evaluate the detector acceptance as a function of energy for different primary particles, simulations have been performed using CORSIKA [22] with QGSJET01 [23] and FLUKA [24] as high- and low-energy hadronic interaction models, respectively. The Monte Carlo showers have been processed through a complete detector simulation and reconstruction chain $[16,25]$. In Fig. 1 we show the energy-dependent relative exposure obtained after trigger, quality cuts, and fiducial volume cuts for primary photons, protons and iron nuclei (normalized to $10 \mathrm{EeV}$ protons). After fiducial volume cuts, the acceptance for photons is close to the acceptance for nuclear primaries. Thus, the relative abundances of photon and nuclear primaries are preserved to a good approximation. In a similar way to Hybrid-1, we apply, for the derivation of an upper limit on the photon fraction, an efficiency correction according to the acceptances after fiducial volume cuts which is conservative and independent of assumptions about the actual primary fluxes (factor " $\epsilon_{\mathrm{fvc}}$ ", see Appendix A).

Applying the selection cuts to the data, there remain $n_{\text {total }}^{\prime}\left(E_{\text {thr }}^{\gamma}\right)=2063,1021,436$ and 131 events with energies greater than $E_{\text {thr }}^{\gamma}=2,3,5$ and $10 \mathrm{EeV}$, respectively. The label $\gamma$ in $E_{\text {thr }}^{\gamma}$ indicates that the missing energy correction for photons has been applied. To obtain $n_{\text {total }}\left(E_{\text {thr }}^{\gamma}\right)$ from the total number of events $n_{\text {total }}^{\prime}\left(E_{\text {thr }}^{\gamma}\right)$ after fiducial volume cuts, those events need to be rejected where clouds may have disturbed the observation. The presence of clouds could change the efficiencies which are shown in Fig. 1. Also, the reconstructed $X_{\max }$ values may be affected. Particularly, clouds may obscure early parts of the shower profile such that the remaining event profile looks deeply penetrating and, hence, photon-like. Therefore we only use data where any disturbance by clouds can be excluded using information from the IR cloud monitoring cameras [26,27]. In Hybrid-1 all events were individually checked. As this is hardly feasible for the events in the present data set (a full automatic processing of cloud data is in preparation), the following approach is adopted. To determine the efficiency $\epsilon_{\mathrm{clc}}$ of passing the cloud cut we used the sample of events with energy above $10 \mathrm{EeV}$. Accepting only events where any disturbance by clouds could be excluded, 67 events out of 131 have been selected, corresponding to $\epsilon_{\mathrm{clc}} \simeq 0.51$. We 

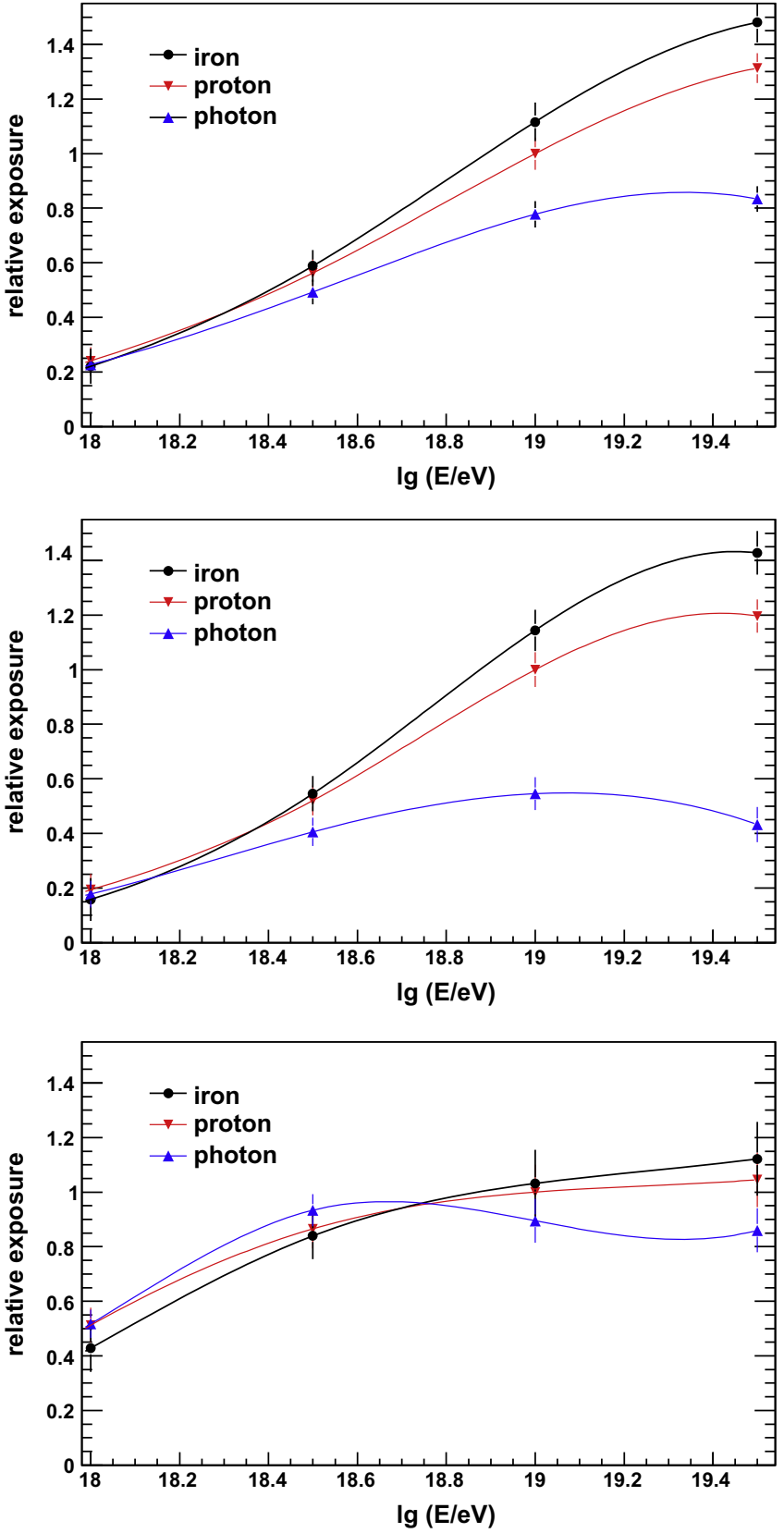

Fig. 1. Relative exposure to primary photons, protons and iron nuclei, normalized to protons at $10 \mathrm{EeV}$. Top panel requiring hybrid trigger, center panel after applying quality cuts, bottom panel after applying fiducial volume cuts (see text). In order to guide the eye polynomial fits are superimposed to the obtained values.

confirmed that this efficiency also holds at lower energy by applying the same criteria to a sub-set of $\sim 300$ events at $\sim 3 \mathrm{EeV}$. The final number of $n_{\text {total }}\left(E_{\mathrm{thr}}^{\gamma}\right)$ is then given by $n_{\text {total }}\left(E_{\mathrm{thr}}^{\gamma}\right)=\epsilon_{\mathrm{clc}}$. $n_{\text {total }}^{\prime}\left(E_{\text {thr }}^{\gamma}\right)$.

As the present data set above $2 \mathrm{EeV}$ is about a factor $\sim 15$ larger than the one used in Hybrid-1, a different statistical method is applied to derive the photon limit. For the derivation of the limit in Hybrid-1, each selected event was individually compared with high-statistics photon simulation, using the respective primary energy and direction as simulation input. This method is CPU demanding, and tailormade for a relatively small number of events. We therefore adopt for our analysis the method applied in Ref. [2] which needs as an input the total number of events, the number of photon candidates (events having "photon-like" characteristics, see below) and proper correction factors accounting for inefficiencies. The $95 \%$ c.l. upper limit $F_{\gamma}^{95}\left(E_{\mathrm{thr}}\right)$ on the fraction of photons in the cosmic-ray flux above $E_{\mathrm{thr}}$ is then given by

$F_{\gamma}^{95}\left(E_{\text {thr }}\right)=\frac{n_{\gamma \text {-cand }}^{95}\left(E_{\text {thr }}^{\gamma}\right)}{n_{\text {total }}\left(E_{\text {thr }}^{\gamma}\right)}$,

where $n_{\gamma \text {-cand }}^{95}$ is the $95 \%$ c.l. upper limit on the number of photon candidates and $n_{\text {total }}$ the total number of selected events. As it is not known in advance whether photons indeed compose only a negligible fraction of the cosmic-ray flux, we apply the missing energy correction appropriate for photons to all events and take here $n_{\text {total }}\left(E_{\text {thr }}^{\gamma}\right)$. This is conservative (larger value of $\left.F_{\gamma}^{95}\right)$, since using the missing energy correction for hadrons (factor $\simeq 1.07-1.14[28,21]$ ) would increase the total number of events above $E_{\mathrm{thr}}$, i.e. $n_{\text {total }}\left(E_{\text {thr }}^{\gamma}\right)<n_{\text {total }}\left(E_{\text {thr }}^{\text {had }}\right)$.

A scatter plot of $X_{\max }$ vs. energy for all events above $E_{\mathrm{thr}}^{\gamma}=2 \mathrm{EeV}$ with $X_{\max } \geqslant 800 \mathrm{~g} \mathrm{~cm}^{-2}$ surviving quality, fiducial volume and cloud cuts is shown in Fig. 2. Statistical uncertainties in individual events are typically a few percent in energy and $\sim 15-30 \mathrm{~g} \mathrm{~cm}^{-2}$ in $X_{\text {max }}$. Systematic uncertainties are $\sim 22 \%$ in energy [29] and $\sim 11 \mathrm{~g} \mathrm{~cm}^{-2}$ in $X_{\max }[15]$.

The upper limit on the number of photon candidates $n_{\gamma \text {-cand }}^{95}$ is given by $n_{\gamma \text {-cand }}^{95}=n_{\gamma \text {-cand.obs }}^{95} / \epsilon_{\text {obs }}$, where $n_{\gamma \text {-cand.obs }}^{95}$ is the $95 \%$ c.l. upper limit on the number of photon candidates $n_{\gamma \text {-cand,obs }}$ extracted ("observed") from the data set and $\epsilon_{\mathrm{obs}}$ is the corresponding efficiency. $n_{\gamma \text {-cand.obs }}$ is taken as the number of events which have the observed $X_{\max }$ above the median $X_{\max }^{\gamma \text { med }}$ of the distribution expected for photons of that energy and direction ("photon candidate cut"). Additionally, on these particular events individual cloud checks have been performed, and only events that pass this cloud check are finally considered as photon candidates. In Fig. 2, typical values of $X_{\max }^{\gamma, \text { med }}(E)$ are indicated as a function of energy (solid red line). To extract the specific value of $X_{\max }^{\gamma, \operatorname{med}}$ for each individual event,

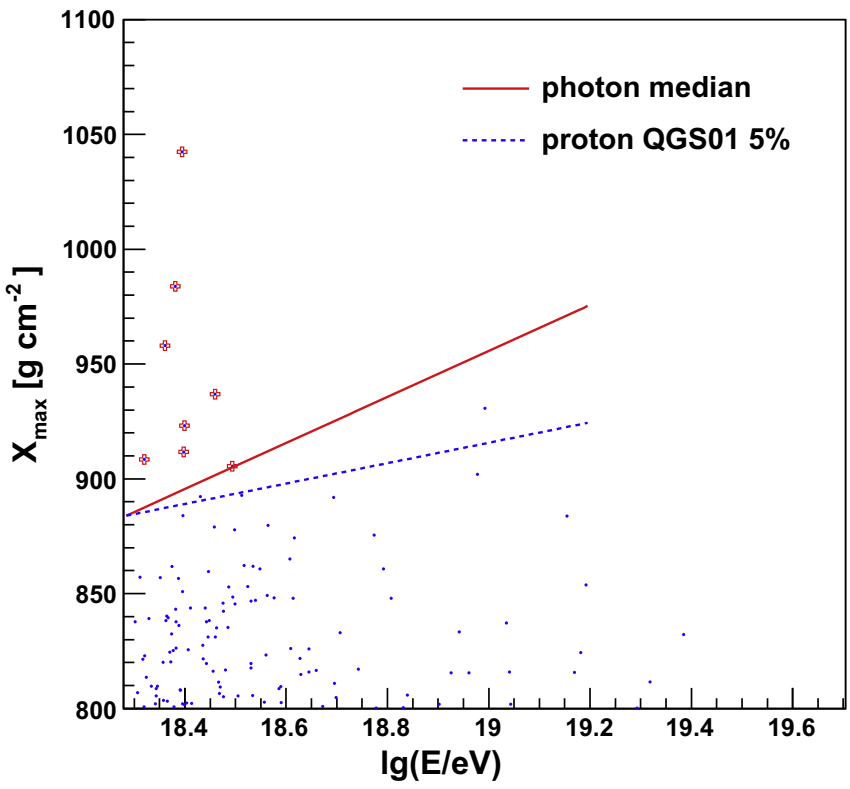

Fig. 2. Closeup of the scatter plot of $X_{\max }$ vs. energy for all events (blue dots) with $X_{\max }$ above $800 \mathrm{~g} \mathrm{~cm}^{-2}$ and energy above $2 \mathrm{EeV}$, after quality, fiducial volume and cloud cuts. Red crosses show the 8 photon candidate events (see text). The solid red line indicates the typical median depth of shower maximum for primary photons, parameterized as $X_{\max }^{\gamma, \operatorname{med}}=a \cdot y+b$, for $y=\lg (E / \mathrm{EeV}), y=[0,1.2]$, where $a=100 \mathrm{~g} \mathrm{~cm}^{-2}$ and $b=856 \mathrm{~g} \mathrm{~cm}^{-2}$. The dashed blue line results from simulations of primary protons using QGSJET01. A fraction of 5\% of the simulated proton showers had $X_{\max }$ values larger than indicated by the line. (For interpretation of the references to colour in this figure legend, the reader is referred to the web version of this article.) 
Table 1

Characteristic parameters for the eight events surviving the photon candidate cut ( $\Delta X_{\max }$ refers to the statistical uncertainty).

\begin{tabular}{lcll}
\hline id & $X_{\max }\left(\mathrm{g} \mathrm{cm}^{-2}\right)$ & $\Delta X_{\max }\left(\mathrm{g} \mathrm{cm}^{-2}\right)$ & $E_{\gamma}(\mathrm{EeV})$ \\
\hline 2051232 & 923 & 17 & 2.5 \\
2053796 & 905 & 32 & 3.1 \\
2201129 & 958 & 29 & 2.3 \\
2566058 & 908 & 20 & 2.1 \\
2798252 & 937 & 29 & 2.9 \\
3478238 & 984 & 12 & 2.4 \\
3554364 & 1042 & 12 & 2.5 \\
3690306 & 912 & 27 & 2.5 \\
\hline
\end{tabular}

dedicated simulations with primary photons have been performed for all potential candidate events, assuming the corresponding energy and geometry.

There are $n_{\gamma \text {-cand,obs }}=8,1,0,0$ photon candidate events with energies greater than 2, 3, 5 and $10 \mathrm{EeV}$, respectively. These candidate events are marked by red crosses in Fig. 2 and the event parameters are listed in Table 1. As an illustration, the shower profile of the candidate with the deepest $X_{\max }$ is displayed in the left panel of Fig. 3 ; in the right panel the measured $X_{\max }$ value is shown along with the results of the dedicated photon simulations.

We checked with simulations whether the observed number of photon candidate events is significantly larger than the expectation in case of nuclear primaries only, i.e. whether primary photons appear to be required to explain the photon candidates. The quantitative estimation of the background expected from nuclear primaries suffers from substantial uncertainties, namely the uncertainty of the primary composition in this energy range (a larger background to photons would originate from lighter nuclear primaries) and the uncertainty in the high energy hadronic interactions models (for instance, reducing the proton-air cross-section allows proton primaries to penetrate deeper into the atmosphere). From simulations using QGSJET01 as the hadronic interaction model, we found that the observed number of photon candidate events is well within the number of background events expected from a pure proton and a pure iron composition. For energies larger than $2 \mathrm{EeV}$ about 30 events are expected in the analyzed time window for proton and 0.3 for iron. The corresponding numbers above $3,5,10 \mathrm{EeV}$ are about $12,4,1$ events for proton and about $0.2,0.1$, 0.0 events for iron. Scenarios of a mixed composition, as also favored by our results on $\left\langle X_{\max }\right\rangle$ [15], can reproduce the observation.
We conclude that the observed photon candidate events may well be due to nuclear primaries only. This also holds for the candidate event with the largest $X_{\max }$ shown in Fig. 3: proton showers with comparable or larger $X_{\max }$ value occur at a level of a few out of thousand simulated events.

We now continue to derive the upper limit to the photon fraction. $n_{\gamma \text {-cand,obs }}^{95}$ is calculated from $n_{\gamma \text {-cand,obs }}$ using the Poisson distribution and assuming no background, i.e. $n_{\gamma \text {-cand,obs }}$ is not reduced by subtracting any event that may actually be due to nuclear primaries. This procedure represents the most conservative approach as it maximizes the value of $n_{\gamma \text {-cand.obs. }}^{95}$ The efficiency $\epsilon_{\mathrm{obs}}$ of photons passing all cuts is given by $\epsilon_{\mathrm{obs}}=\epsilon_{\mathrm{fvc}} \epsilon_{\mathrm{pcc}}$ where $\epsilon_{\mathrm{fvc}} \simeq 0.72-0.77$ (see Table 2) comes from the acceptance after fiducial volume cuts (see Appendix) and, by construction, $\epsilon_{\mathrm{pcc}}=0.50$ is given by the photon candidate cut above the median of the $X_{\max }$ distribution for photons. Thus, the upper limit is calculated according to

$F_{\gamma}^{95}\left(E_{\text {thr }}\right)=\frac{n_{\gamma-\text { cand }, \text { obs }}^{95}\left(E_{\text {thr }}^{\gamma}\right) \frac{1}{\epsilon_{\text {fvc }}} \frac{1}{\epsilon_{\mathrm{pcc}}}}{n_{\text {total }}^{\prime}\left(E_{\text {thr }}^{\gamma}\right) \epsilon_{\mathrm{clc}}}$.

Applied to the data, upper limits of 3.8\%, 2.4\%, 3.5\% and $11.7 \%$ on the fraction of cosmic-ray photons above 2, 3, 5 and $10 \mathrm{EeV}$ are obtained at $95 \%$ c.l.. Table 2 provides a summary of the quantities used in the derivation of the integral upper limits.

We studied the robustness of the results against different sources of uncertainty. Varying individual event parameters or the selection criteria, within the experimental resolution, leaves the results essentially unchanged. Uncertainties in the determination of the efficiency factors used in Eq. (2) are estimated to correspond to an uncertainty $\Delta F_{\gamma}^{95} / F_{\gamma}^{95} \simeq 0.15$. Increasing (reducing) all reconstructed $X_{\max }$ values by $\Delta X_{\max }^{\text {syst }}=11 \mathrm{~g} \mathrm{~cm}^{-2}$ [15] changes the number of photon candidates above $2 \mathrm{EeV}$ by $+1( \pm 0)$ and above $3 \mathrm{EeV}$ by $\pm 0(-1)$, while it does not affect the higher energies. The limits then become $4.1 \%$ (3.8\%) above $2 \mathrm{EeV}$ and $2.4 \%(1.5 \%)$ above $3 \mathrm{EeV}$. The energy scale $E_{\text {thr }}$ which the limit $F_{\gamma}^{95}\left(E_{\mathrm{thr}}\right)$ refers to, has a $22 \%$ systematic uncertainty [29]. Hence, the numerical values of the limits $F_{\gamma}^{95}$ derived here refer to an effective energy threshold $E_{\mathrm{thr}}^{\text {eff }}=k_{E} \times E_{\mathrm{thr}}$, with $k_{E}=0.78 \ldots 1.22$. Related to an increase (reduction) of the energy scale is a small upward (downward) shift of the $X_{\max }$ value used for the photon candidate cut, leading to stronger (weaker) criteria for an event to pass this cut. This shift amounts to $\sim 7 \mathrm{~g} \mathrm{~cm}^{-2}$ for a $22 \%$ change of the energy scale. Finally, an uncertainty $<10 \mathrm{~g} \mathrm{~cm}^{-2}$ on the simulated photon $X_{\max }$ values comes from the need to extrapolate the photonuclear
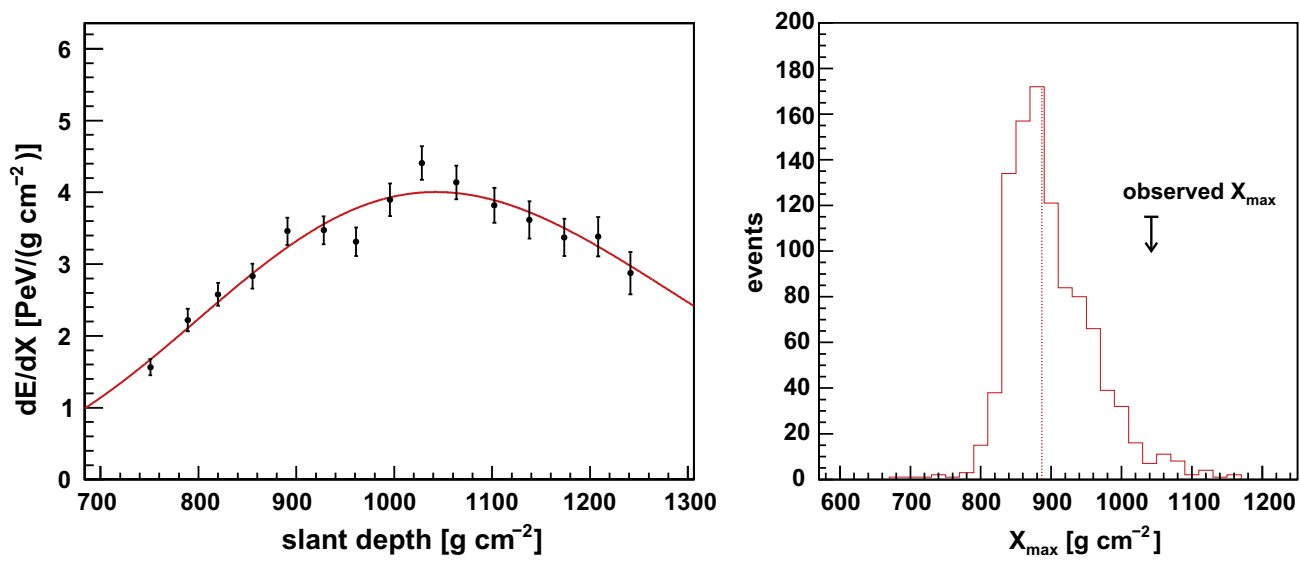

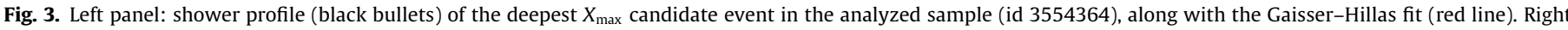

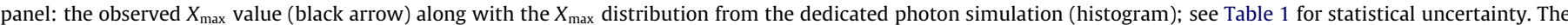

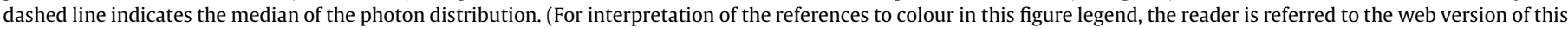
article.) 
Table 2

Summary of the quantities used in the derivation of the integral upper limits on the photon fraction for $E_{\mathrm{thr}}^{\gamma}=2,3,5$, and $10 \mathrm{EeV}$. Not listed are the efficiencies $\epsilon_{\mathrm{clc}}=0.51$ and $\epsilon_{\mathrm{pcc}}=0.50$ which do not depend on $E_{\mathrm{thr}}^{\gamma}$.

\begin{tabular}{rllllc}
\hline$E_{\text {thr }}^{\gamma}(\mathrm{EeV})$ & $n_{\gamma \text {-cand,obs }}$ & $n_{\gamma \text {-cand,obs }}^{95}$ & $n_{\text {total }}^{\prime}$ & $\epsilon_{\mathrm{fvc}}$ & $F_{\gamma}^{95}(\%)$ \\
\hline 2 & 8 & 14.44 & 2063 & 0.72 & 3.8 \\
3 & 1 & 4.75 & 1021 & 0.77 & 2.4 \\
5 & 0 & 3.0 & 436 & 0.77 & 3.5 \\
10 & 0 & 3.0 & 131 & 0.77 & 11.7 \\
\hline
\end{tabular}

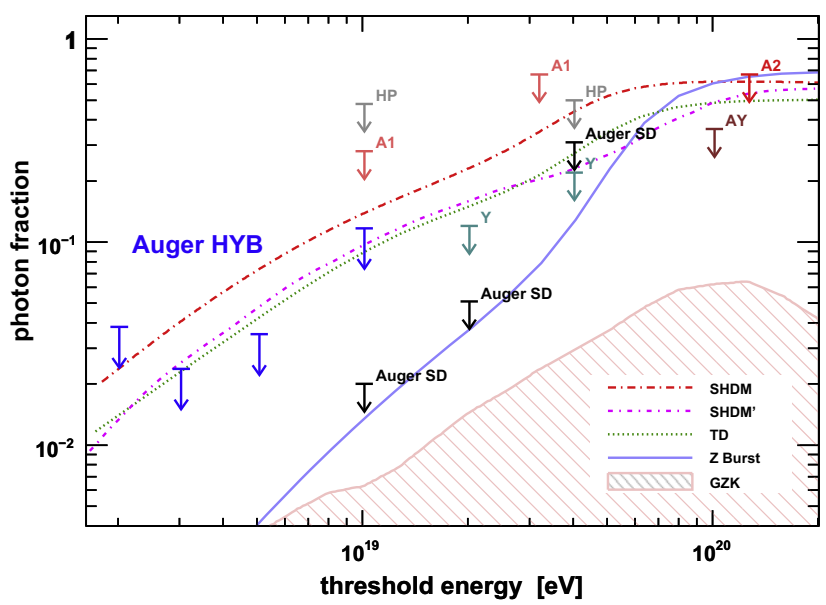

Fig. 4. Upper limits on the photon fraction in the integral cosmic-ray flux for different experiments: AGASA (A1, A2) [3,4], AGASA-Yakutsk (AY) [31], Yakutsk (Y) [32], Haverah Park (HP) [5,6]. In black the limits from the Auger surface detector (Auger SD) [2] and in blue the limits above 2, 3, 5, and $10 \mathrm{EeV}$ derived in this work (Auger HYB). The shaded region shows the expected GZK photon fraction as derived in [7]. Lines indicate predictions from top-down models, see [8,33] and [34]. (For interpretation of the references to colour in this figure legend, the reader is referred to the web version of this article.)

cross-section to high energy [30]. Adding in quadrature the discussed uncertainties in $X_{\max }$ gives an effective total uncertainty of $\sim 16 \mathrm{~g} \mathrm{~cm}^{-2}$. Increasing (reducing) all reconstructed $X_{\max }$ values by this amount changes the number of photon candidates above 2 and $3 \mathrm{EeV}$ by $+3( \pm 0)$ and by $+1(-1)$. Accordingly the limits then become $4.8 \%$ (3.8\%) above $2 \mathrm{EeV}$ and 3.1\% (1.5\%) above $3 \mathrm{EeV}$, while the limits above 5 and $10 \mathrm{EeV}$ are unchanged.

\section{Discussion}

The derived upper limits are shown in Fig. 4 along with previous experimental limits and model predictions (see Ref. [34] for a review and references). These new bounds are the first ones at energies below $10 \mathrm{EeV}$ and, together with Hybrid-1, the only ones obtained so far from fluorescence observations (all other limits coming from ground arrays). The results complement the previous constraints on top-down models from Auger surface detector data. It should be noted that due to the steep flux spectrum, even the previous Auger bound of $2 \%$ above $10 \mathrm{EeV}$ only marginally constrains the photon contribution above lower threshold energies (for instance, even above $5 \mathrm{EeV}, \sim 75 \%$ of the events are in the previously untested energy range of 5-10 EeV).

The photon limits derived in this work also help to reduce certain systematic uncertainties in other analyses of air shower data such as (i) energy spectrum: the Auger method of reconstructing the energy spectrum does not suffer from a large contamination from photons at EeV energies; (ii) nuclear primary composition: the interpretation of observables sensitive to the primary particle (for instance the observed average $X_{\max }$ ) in terms of a nuclear pri- mary composition can only be marginally biased by contributions from photons; (iii) proton-air cross-section: the possible contamination from photons was one of the dominant uncertainties for deriving the proton-air cross-section $[13,14]$, and this uncertainty is now significantly reduced (to $\sim 50 \mathrm{mb}$ for data at $\mathrm{EeV}$ energies, which corresponds to a relative uncertainty of $\sim 10 \%$ ).

In future photon searches, the separation power between photons and nuclear primaries can be enhanced by adding the detailed information measured with the surface detectors in hybrid events. For an estimate of the future sensitivity of Auger to photons see Ref. [34]. The information on event directions can also be used in future analyses; for instance, an excess flux of photons from the direction of the galactic center (e.g. Ref. [10]) can be searched for.

\section{Acknowledgements}

The successful installation and commissioning of the Pierre Auger Observatory would not have been possible without the strong commitment and effort from the technical and administrative staff in Malargüe.

We are very grateful to the following agencies and organizations for financial support: Comisión Nacional de Energía Atómica, Fundación Antorchas, Gobierno De La Provincia de Mendoza, Municipalidad de Malargüe, NDM Holdings and Valle Las Leñas, in gratitude for their continuing cooperation over land access, Argentina; the Australian Research Council; Conselho Nacional de Desenvolvimento Científico e Tecnológico (CNPq), Financiadora de Estudos e Projetos (FINEP), Fundação de Amparo à Pesquisa do Estado de Rio de Janeiro (FAPERJ), Fundação de Amparo à Pesquisa do Estado de São Paulo (FAPESP), Ministério de Ciência e Tecnologia (MCT), Brazil; AVCR AV0Z10100502 and AV0Z10100522, GAAV KJB300100801 and KJB100100904, MSMT-CR LA08016, LC527, 1M06002, and MSM0021620859, Czech Republic; Centre de Calcul IN2P3/CNRS, Centre National de la Recherche Scientifique (CNRS), Conseil Régional Ile-de-France, Département Physique Nucléaire et Corpusculaire (PNC-IN2P3/CNRS), Département Sciences de l'Univers (SDU-INSU/CNRS), France; Bundesministerium für Bildung und Forschung (BMBF), Deutsche Forschungsgemeinschaft (DFG), Finanzministerium Baden-Württemberg, HelmholtzGemeinschaft Deutscher Forschungszentren (HGF), Ministerium für Wissenschaft und Forschung, Nordrhein-Westfalen, Ministerium für Wissenschaft, Forschung und Kunst, Baden-Württemberg, Germany; Istituto Nazionale di Fisica Nucleare (INFN), Ministero dell'Istruzione, dell'Università e della Ricerca (MIUR), Italy; Consejo Nacional de Ciencia y Tecnología (CONACYT), Mexico; Ministerie van Onderwijs, Cultuur en Wetenschap, Nederlandse Organisatie voor Wetenschappelijk Onderzoek (NWO), Stichting voor Fundamenteel Onderzoek der Materie (FOM), Netherlands; Ministry of Science and Higher Education, Grant Nos. 1 P03 D 014 30, N202 090 31/0623, and PAP/218/2006, Poland; Fundação para a Ciência e a Tecnologia, Portugal; Ministry for Higher Education, Science, and Technology, Slovenian Research Agency, Slovenia; Comunidad de Madrid, Consejería de Educación de la Comunidad de Castilla La Mancha, FEDER funds, Ministerio de Ciencia e Innovación, Xunta de Galicia, Spain; Science and Technology Facilities Council, United Kingdom; Department of Energy, Contract No. DE-AC0207CH11359, National Science Foundation, Grant No. 0450696, The Grainger Foundation USA; ALFA-EC/ HELEN, European Union 6th Framework Program, Grant No. MEIF-CT-2005-025057, and UNESCO.

\section{Appendix A. Acceptance correction}

The fraction of photons $f_{\gamma}$ in the cosmic-ray flux integrated above an energy threshold $E_{\mathrm{thr}}$ is given by 
$f_{\gamma}\left(E \geqslant E_{\mathrm{thr}}\right)=\frac{\int_{E_{\mathrm{thr}}} \Phi_{\gamma}(E) d E}{\int_{\mathrm{E}_{\mathrm{thr}}} \Phi_{\gamma}(E) d E+\sum_{i} \int_{E_{\mathrm{thr}}} \Phi_{i}(E) d E}$

where $\Phi_{\gamma}(E)$ denotes the differential flux of photons and $\Phi_{i}(E), i=\mathrm{p}, \mathrm{He}, \ldots$ the fluxes of nuclear primaries. by

The fraction of photons $f_{\gamma}^{\text {det }}$ as registered by the detector is given

$f_{\gamma}^{\mathrm{det}}\left(E \geqslant E_{\mathrm{thr}}\right)=\frac{\int_{E_{\mathrm{thr}}} A_{\gamma}(E) \Phi_{\gamma}(E) d E}{\int_{E_{\mathrm{thr}}} A_{\gamma}(E) \Phi_{\gamma}(E) d E+\sum_{i} \int_{E_{i}} A_{i}(E) \Phi_{i}(E) d E}$

with $A_{\gamma}(E)$ and $A_{i}(E)$ being the detector acceptances to photons and nuclear primaries, respectively. $E_{i}$ denotes the effective threshold energy for primary nucleus $i$.

Thus, the upper limit $f_{\gamma}^{\text {ul,det }}$ obtained to the registered data, $f_{\gamma}^{\text {uldet }}>f_{\gamma}^{\text {det }}$, needs to be corrected to resemble an upper limit on the fraction of photons in the cosmic-ray flux. For the present analysis, a conservative and model-independent correction is applied as follows. The approach adopted here extends the one introduced in Hybrid-1, as we now also treat the case of $A_{\gamma}(E) \neq$ const.

$E_{\mathrm{thr}}$ corresponds to the analysis threshold energy assuming primary photons. $E_{i}$ is related to $E_{\mathrm{thr}}$ by the ratios of the missing energy corrections $m_{\gamma}$ (for photons) and $m_{i}$ (for nuclear primaries),

$E_{i}=E_{\mathrm{thr}} \cdot \frac{m_{i}}{m_{\gamma}}$

Since $m_{\gamma} \simeq 1.01$ [21] and $m_{i} \simeq 1.07-1.14$ [28], $E_{i}>E_{\mathrm{thr}}$. Thus, replacing $E_{i}$ by $E_{\text {thr }}$,

$$
\begin{aligned}
f_{\gamma}^{\mathrm{det}}\left(E \geqslant E_{\mathrm{thr}}\right)>\frac{\int_{E_{\mathrm{thr}}} A_{\gamma}(E) \Phi_{\gamma}(E) d E}{\int_{E_{\mathrm{thr}}} A_{\gamma}(E) \Phi_{\gamma}(E) d E+\sum_{i} \int_{E_{\mathrm{thr}}} A_{i}(E) \Phi_{i}(E) d E} \\
\geqslant \frac{\int_{E_{\mathrm{thr}}} A_{\gamma}^{\mathrm{min}} \Phi_{\gamma}(E) d E}{\int_{E_{\mathrm{thr}}} A_{\gamma}^{\mathrm{min}} \Phi_{\gamma}(E) d E+\sum_{i} \int_{E_{\mathrm{thr}}} A_{i}(E) \Phi_{i}(E) d E},
\end{aligned}
$$

where $A_{\gamma}^{\min }$ refers to the minimum value of $A_{\gamma}\left(E \geqslant E_{\text {thr }}\right)$ and using $a /(a+b) \geqslant a^{\prime} /\left(a^{\prime}+b\right)$ for $a \geqslant a^{\prime} \geqslant 0$ and $b>0$.

Next, the acceptance ratio $\epsilon_{i}(E)=A_{\gamma}^{\min } / A_{i}(E)$ is introduced,

$f_{\gamma}^{\mathrm{det}}\left(E \geqslant E_{\mathrm{thr}}\right)>\frac{\int_{E_{\mathrm{thr}}} A_{\gamma}^{\mathrm{min}} \Phi_{\gamma}(E) d E}{\int_{E_{\mathrm{thr}}} A_{\gamma}^{\min } \Phi_{\gamma}(E) d E+\sum_{i} \int_{E_{\mathrm{thr}}} \frac{A_{\gamma}^{\min }}{\epsilon_{i}(E)} \Phi_{i}(E) d E}$.

From Fig. 1 the minimum acceptance ratio $\epsilon_{\min }\left(E_{\mathrm{thr}}\right) \leqslant \epsilon_{i}\left(E \geqslant E_{\mathrm{thr}}\right)$ can be extracted for each threshold energy $E_{\mathrm{thr}}$. In the current analysis, $\quad \epsilon_{\min }\left(E_{\text {thr }}\right) \equiv \epsilon_{\text {fvc }}\left(E_{\text {thr }}\right) \simeq 0.72,0.77,0.77,0.77 \quad$ for $E_{\mathrm{thr}}=2,3,5,10 \mathrm{EeV}$. Hence, it follows:

$$
\begin{aligned}
f_{\gamma}^{\mathrm{det}}(E & \left.\geqslant E_{\mathrm{thr}}\right)>\frac{\int_{E_{\mathrm{thr}}} \Phi_{\gamma}(E) d E}{\int_{E_{\mathrm{thr}}} \Phi_{\gamma}(E) d E+\frac{1}{\epsilon_{\mathrm{fvc}}\left(E_{\mathrm{thr}}\right)} \sum_{i} \int_{E_{\mathrm{thr}}} \Phi_{i}(E) d E} \\
& >\epsilon_{\mathrm{fvc}}\left(E_{\mathrm{thr}}\right) \cdot \frac{\int_{E_{\mathrm{thr}}} \Phi_{\gamma}(E) d E}{\int_{E_{\mathrm{thr}}} \Phi_{\gamma}(E) d E+\sum_{i} \int_{E_{\mathrm{thr}}} \Phi_{i}(E) d E} \\
& =\epsilon_{\mathrm{fvc}}\left(E_{\mathrm{thr}}\right) \cdot f_{\gamma}\left(E \geqslant E_{\mathrm{thr}}\right),
\end{aligned}
$$

where it was used that $\frac{1}{\epsilon_{\mathrm{fvc}}\left(E_{\mathrm{thr}}\right)}>1$.

Consequently, an upper limit $F_{\gamma}^{\mathrm{ul}}$ to the fraction of photons in the cosmic-ray flux can conservatively be calculated as
$F_{\gamma}^{\mathrm{ul}}=f_{\gamma}^{\mathrm{ul}, \mathrm{det}} / \epsilon_{\mathrm{fvc}}>f_{\gamma}^{\mathrm{det}} / \epsilon_{\mathrm{fvc}}>f_{\gamma}$.

The upper limit obtained this way does not depend on assumptions about the differential fluxes $\Phi_{\gamma}(E)$ and $\Phi_{i}(E)$.

\section{References}

[1] J. Abraham et al., Pierre Auger Collaboration, Astropart. Phys. 27 (2007) 155. astro-ph/0606619.

[2] J. Abraham et al., Pierre Auger Collaboration, Astropart. Phys. 29 (2008) 243. arXiv:0712.1147 [astro-ph].

[3] K. Shinozaki et al., Astrophys. J. 571 (2002) L117-L120.

[4] M. Risse et al., Phys. Rev. Lett. 95 (2005) 171102.

[5] M. Ave, J.A. Hinton, R.A. Vazquez, A.A. Watson, E. Zas, Phys. Rev. Lett. 85 (2000) 2244.

[6] M. Ave, J.A. Hinton, R.A. Vazquez, A.A. Watson, E. Zas, Phys. Rev. D 65 (2002) 063007.

[7] G. Gelmini, O. Kalashev, D.V. Semikoz, JCAP 11 (2007). arXiv:0706.2181 [astroph].

[8] G. Gelmini, O. Kalashev, D.V. Semikoz, J. Exp. Theor. Phys. 106 (2008) 1061 1082. astro-ph/050612.

[9] G. Sigl, Phys. Rev. D 75 (2007) 103001. astro-ph/0703403.

[10] A. Kusenko, J. Schissel, F.W. Stecker, Astropart. Phys. 25 (2006) 242-245. astro$\mathrm{ph} / 0508142$

[11] P. Bhattacharjee, G. Sigl, Phys. Rep. 327 (2000) 109-247. astro-ph/9811011.

[12] N. Busca, D. Hooper, E.W. Kolb, Phys. Rev. D 73 (2006) 123001. astro-ph/ 0603055.

[13] K. Belov, HiRes Collaboration, Nucl. Phys. B - Proc. Suppl. 151 (2006) 197.

[14] R. Ulrich, J. Blümer, R. Engel, F. Schüssler, M. Unger, Nucl. Phys. B - Proc. Suppl. 175 (2008) 121-124. arXiv:0709.1392 [astro-ph].

[15] M. Unger, Pierre Auger Collaboration, in: 30th International Cosmic Ray Conference (ICRC 07), Mérida, Yucatan, Mexico, 3-11 July 2007, vol. 4, 2007, p 373, arXiv:0706.1495 [astro-ph].

[16] S. Argirò, S.L.C. Barroso, J. Gonzalez, L. Nellen, T. Paul, T.A. Porter, L. Prado Jr., M Roth, R. Ulrich, D. Veberič, Nucl. Instrum. Meth. A 580 (2007) 1485-1496. arXiv:0707.1652 [astro-ph].

[17] R. Knapik et al., Pierre Auger Collaboration, in: 30th International Cosmic Ray Conference (ICRC 07), Mérida, Yucatan, Mexico, 3-11 July 2007, vol. 4, 2007, p. 343, arXiv:0708.1924 [astro-ph].

[18] B. Keilhauer et al., Pierre Auger Collaboration, in: 29th International Cosmic Ray Conference (ICRC 05), Pune, India, 3-10 August 2005, vol. 7, 2005, p. 123, astro-ph/0507275.

[19] S.Y. BenZvi et al., Pierre Auger Collaboration, in: 30th International Cosmic Ray Conference (ICRC 07), Mérida, Yucatan, Mexico, 3-11 July 2007, vol. 4, 2007, p. 355, arXiv:0706.3236 [astro-ph]

[20] M. Unger, B.R. Dawson, R. Engel, F. Schüssler, R. Ulrich, Nucl. Instrum. Meth. A 588 (2008) 433-441.

[21] T. Pierog, R. Engel, D. Heck, Czech. J. Phys. 56 (2006) A161-A172. astro-ph/ 0602190.

[22] D. Heck et al., Report FZKA 6019, 6097, Forschungzentrum Karlsruhe, 1998

[23] N.N. Kalmykov, S.S. Ostapchenko, Phys. Atom. Nucl. 56 (1993) 346-353.

[24] A. Fassò, A. Ferrari, J. Ranft, B.E. Sala, CERN-2005-10, INFN/TC_05/11, SLAC-R773.

[25] L. Prado Jr. et al., Nucl. Instrum. Meth. A 545 (2005) 632-642.

[26] R. Cester et al., in: 29th International Cosmic Ray Conference (ICRC 05), Pune, India, 3-10 August 2005, vol. 8, 2005, p. 347

[27] B. Fick et al., JINST 1 (2006) P11003. astro-ph/0507334

[28] H.M.J. Barbosa, F. Catalani, J.A. Chinellato, C. Dobrigkeit, Astropart. Phys. 22 (2004) 159-166. astro-ph/0310234.

[29] J. Abraham et al., Pierre Auger Collaboration, Phys. Rev. Lett. 101 (2008) 061101. arXiv:0806.4302 [astro-ph].

[30] M. Risse et al., Czech. J. Phys. A 56 (2006) 327. astro-ph/0512434.

[31] G. Rubtsov et al., Phys. Rev. D 73 (2006) 063009. astro-ph/0601449.

[32] A.V. Glushkov et al., JETP Lett. 85 (2007) 163. astro-ph/0701245.

[33] J. Ellis, V.E. Mayes, D.V. Nanopoulos, Phys. Rev. D 74 (2006) 115003. astro-ph/ 0512303.

[34] M. Risse, P. Homola, Mod. Phys. Lett. A 22 (2007) 749. astro-ph/0702632. 